九州大学学術情報リポジトリ

Kyushu University Institutional Repository

\title{
Formation-Flying Trajectory Design Based on Attractive Sets of Tschauner-Hempel Equations
}

\section{Yamane, Motoki}

Digital Solutions and Delivery Business Unit, End point Business Division, Solution Development Department

Bando, Ma i

Department of Aeronautics and Astronautics, Kyushu University : Associate Professor

HOKAMOTO, Shinji

Department of Aeronautics and Astronautics, Kyushu University : Professor

ht tp://hdl. hand le. net/2324/4479056

出版情報：Journal of guidance, control, and dynamics. 43 (10), pp. 1943-1951，2020-08-25. American Institute of Aeronautics and Astronautics

バージョン :

権利関係 : 


\title{
Formation Flying Trajectory Design based on Attractive Sets of Tschauner-Hempel Equations
}

\author{
Motoki Yamane* \\ FUJITSU LIMITED, Tokyo 140-0052, Japan \\ Mai Bando ${ }^{\dagger}$ and Shinji Hokamoto \\ Kyushu University, 744 Motooka, Nishi-ku, Fukuoka 819-0395, Japan
}

\section{Introduction}

Spacecraft formation flying (SFF), two or more spacecraft traveling and maneuvering together, is an enabling technology for future space missions which has potential to improve mission flexibility and performance. SFF could replace a single large spacecraft with a number of smaller, less-expensive spacecraft and realize a long baseline that is difficult to realize with a single spacecraft. Therefore various missions are considered such as a telescope with a stereoscopic view and a long focal length, radio wave detection, and laser interferometer. The problems of the formation initialization, stationkeeping and reconfiguration have received significant interest by control researchers. Providing fuel-efficient orbit design techniques to change between different formation geometries is the main focus of the reconfiguration problem [1, 2].

The Hill's equations (also known as the Hill-Clohessy-Wiltshire (HCW) equations) are equations of relative motion of a chaser satellite linearized around a target satellite in a circular orbit [3, 4]. The Hill's equations have periodic solutions that are convenient for formation flying. These solutions are useful for formation flying because no control efforts are needed to maintain them. The rendezvous problem for a target on a circular orbit has been studied extensively in the literature [5]. When a target is in an elliptic orbit, the equations of relative motion of a chaser have periodic coefficients. Then, the Tschauner-Hempel (TH) equations are derived after the linearization of the nonlinear equations. The TH equations also possess periodic solutions which can be used for passive formation flying. The state transition matrix of the TH equations is given in various forms [5]. Yamanaka and Ankersen derived the powerful state transition matrix associated with the TH equations [6]. In this solution, the position and velocity of the chaser depend on an initial true anomaly of the target. Therefore the geometry of the solutions have been extensively studied by some authors [7-9]. In [ [7], the fundamental solutions of the TH equations are interpreted geometrically as generalizations of the well-known solutions of the Hill's equations. The effects of eccentricity on the shape and size of relative orbits were studied in [8]. In [9], the geometry of periodic solutions of the TH equations were used to generate the periodic

\footnotetext{
*Digital Solutions \& Delivery Business Unit, Endpoint Business Division, Solution Development Department, Nissay Aroma Square, 5-37-1, Kamata.

$\dagger$ Associate Professor, Department of Aeronautics and Astronautics, Fukuoka, Member AIAA.

†Professor, Department of Aeronautics and Astronautics, Fukuoka, Member AIAA.
} 
orbit family.

The optimal rendezvous/reconfiguration problem using continuous low-thrust is often treated as the optimal control problem. When the performance index is described by a quadratic function, the optimal rendezvous and reconfiguration problem for the HCW equations or the TH equations becomes a linear quadratic (LQ) optimal control problem [10-[13]. The LQ optimal control is given by solving algebraic Riccati equation (ARE) and differential Riccati equation (DRE) for the HCW equations or the TH equations respectively. The attractive sets of optimal control are defined as level sets of the optimal cost [14]. For the LQ optimal control problem, the optimal cost is expressed as a quadratic function of the initial state and the attractive set for time-invariant system becomes an ellipsoidal set. The attractive set can be used to determine the optimal initial state among possible initial states by exploiting the geometry of the ellipsoid. Thus solutions to the ARE and DRE implicitly contain the geometry of the optimal solution and they can provide additional insight into the optimal control problem. This motivates to study the geometric interpretation of the solutions of the ARE and DRE for formation flying by introducing the concept of attractive set of optimal control. In [15], attractive sets for optimal rendezvous problem along a circular orbit was investigated based on the solution of the ARE. It was shown that the optimal initial positions can be obtained as the tangent point between the largest attractive set inscribed in the periodic orbit and the periodic orbit. Moreover, it was revealed that the attractive set of optimal control reflects the dynamical structure of the motion to reduce the control effort and the cost becomes less sensitive to the initial state on a periodic orbit.

For the TH equations, which is represented by a time-periodic linear system, the attractive set of optimal cost becomes time-periodic and makes it difficult to interpret geometrically. In this paper, a novel geometric method to describe the geometry of the time-periodic solutions of DRE using the attractive set is proposed. Then a formation reconfiguration problem along an elliptic orbit, in which a satellite initially located on a periodic orbit is required to change the formation, is solved exploiting the geometry of attractive set.

\section{Equation of Relative Motion}

\section{A. Tschauner-Hempel equations}

Consider a target satellite along an elliptic orbit of period $T$ given by $\boldsymbol{R}_{0}$ shown in Fig. 四, where $\boldsymbol{R}_{0}$ is the radius vector from the center of the Earth to the target satellite; $\theta$ is the true anomaly of the target satellite. The equations of motion of the target satellite are

$$
\begin{gathered}
\ddot{R}_{0}-R_{0} \dot{\theta}=-\left(\frac{\mu_{e}}{R_{0}^{2}}\right) \\
2 \dot{R}_{0} \dot{\theta}+R_{0} \ddot{\theta}=0
\end{gathered}
$$




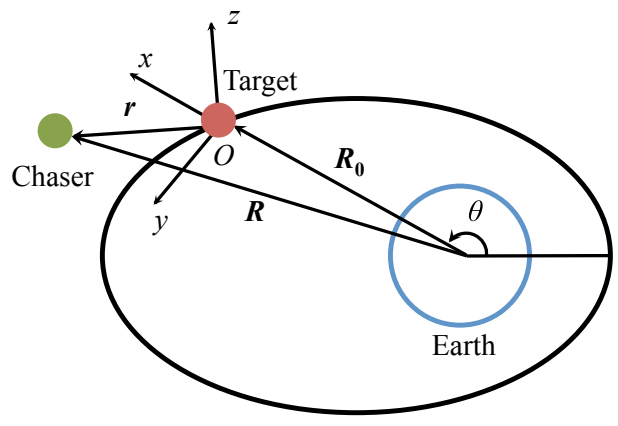

Fig. 1 Target satellite in an elliptic orbit.

where $R_{0}=\left|\boldsymbol{R}_{0}\right|$. Then, the equations of motion of the chaser relative to the target is expressed by

$$
\begin{aligned}
\ddot{x}-2 \dot{\theta} \dot{y}-\ddot{\theta} y-\dot{\theta}^{2} x-\frac{\mu_{e}}{R_{0}^{2}} & =-\frac{\mu_{e}\left(R_{0}+x\right)}{R^{3}} \\
\ddot{y}+2 \dot{\theta} \dot{x}+\ddot{\theta} x-\dot{\theta}^{2} y & =-\frac{\mu_{e} y}{R^{3}} \\
\ddot{z} & =-\frac{\mu_{e} z}{R^{3}}
\end{aligned}
$$

where $R=\left[\left(R_{0}+x\right)^{2}+y^{2}+z^{2}\right]^{\frac{1}{2}}$. The linearization around the target leads to

$$
\begin{aligned}
\ddot{x}-2 \dot{\theta} \dot{y}-\ddot{\theta} y-\left(\dot{\theta}^{2}+2 \frac{\mu_{e}}{R_{0}^{2}}\right) x & =0 \\
\ddot{y}+2 \dot{\theta} \dot{x}+\ddot{\theta} x-\left(\dot{\theta}^{2}-\frac{\mu_{e}}{R_{0}^{2}}\right) y & =0 \\
\ddot{z}+\frac{\mu_{e} z}{R_{0}^{3}} & =0
\end{aligned}
$$

which are referred to as the Tschauner-Hempel equations. In Eqs. (G) - (8)), in-plane motion and out-plane motion are independent and the out-of-plane motion is a simple sinusoidal motion.

In the following, the in-plane motion is considered. Let $\boldsymbol{X}=\left[\begin{array}{cccc}x & y & \dot{x} & \dot{y}\end{array}\right]^{T}$ and $\boldsymbol{u}=\left[\begin{array}{ll}u_{x} & u_{y}\end{array}\right]^{T}$ be the state and control acceleration respectively. Then the state space form of the in-plane motion is denoted by

$$
\dot{\boldsymbol{X}}=A(t) \boldsymbol{X}+B \boldsymbol{u}
$$


where

$$
A=\left[\begin{array}{cccc}
0 & 0 & 1 & 0 \\
0 & 0 & 0 & 1 \\
\dot{\theta}^{2}+2 \frac{\mu_{e}}{R_{0}^{3}} & -\frac{2 \dot{R}_{0} \dot{\theta}}{R_{0}} & 0 & 2 \dot{\theta} \\
\frac{2 \dot{R}_{0} \dot{\theta}}{R_{0}} & \dot{\theta}^{2}-\frac{\mu_{e}}{R_{0}^{3}} & -2 \dot{\theta} & 0
\end{array}\right], B=\left[\begin{array}{ll}
0 & 0 \\
0 & 0 \\
1 & 0 \\
0 & 1
\end{array}\right]
$$

Let the initial time be $t_{0}$. Then, for $0 \leq t_{0} \leq T$, the solution of Eq. (Qd) with $\boldsymbol{u}=0$ is given by

$$
\begin{aligned}
\boldsymbol{X}(t) & =\left[\begin{array}{cccc}
0 & -\frac{s}{\rho} & -\frac{c}{\rho} & -\frac{1}{\rho}(2-3 e s G) \\
\frac{1}{\rho} & -\frac{c}{\rho}\left(1+\frac{1}{\rho}\right) & \frac{s}{\rho}\left(1+\frac{1}{\rho}\right) & 3 \rho G \\
0 & -\frac{\dot{\theta}}{\rho} c & \frac{\dot{\theta}}{\rho} s & \frac{\dot{\theta}}{\rho} e\left(3 c G+\frac{s}{\rho^{2}}\right) \\
\frac{e s \dot{\theta}}{\rho^{3}} & \frac{s \dot{\theta}}{\rho^{2}}\left[2+e \frac{c}{\rho}\left(1-\frac{1}{\rho}\right)\right] & \frac{\dot{\theta}}{\rho}\left[c\left(1+\frac{1}{\rho}\right)+e \frac{s^{2}}{\rho^{3}}\right] & 3 \frac{\dot{\theta}}{\rho}(1-e s G)
\end{array}\right] \boldsymbol{K} \\
& \equiv \Phi_{\theta} \boldsymbol{K}
\end{aligned}
$$

where $\boldsymbol{K}=\left[\begin{array}{llll}K_{1} & K_{2} & K_{3} & K_{4}\end{array}\right]^{\mathrm{T}}, \rho=1+e \cos \theta, c=\rho \cos \theta, s=\rho \sin \theta$,

$$
G(\theta)=\frac{h^{\frac{3}{2}}}{p}\left(t-t_{0}\right)=\int_{\theta\left(t_{0}\right)}^{\theta(t)} \frac{1}{\rho(\tau)^{2}} d \tau
$$

and $h$ is angular momentum of the elliptic orbit [6, [3]. The parameter vector $\boldsymbol{K}$ is uniquely determined by initial state

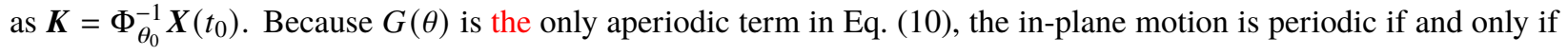
$K_{4}=0$. In this case

$$
\begin{aligned}
& x(t)=-K_{2} \sin \theta-K_{3} \cos \theta \\
& y(t)=\left(\frac{1}{\rho}\right) K_{1}-\left(\frac{c}{\rho}\right)\left[1+\left(\frac{1}{\rho}\right)\right] K_{2}+\left(\frac{s}{\rho}\right)\left[1+\left(\frac{1}{\rho}\right)\right] K_{3}
\end{aligned}
$$

and its implicit function formula is

$$
\frac{x^{2}}{K_{2}^{2}+K_{3}^{2}}+\frac{\left[y-\left(\frac{K_{1}}{\rho}\right)\right]^{2}}{\left[1+\left(\frac{1}{\rho}\right)\right]^{2}\left(K_{2}^{2}+K_{3}^{2}\right)}=1
$$

To understand the geometry of periodic orbits, size and phase parameters are introduced. Define $a=\sqrt{K_{2}^{2}+K_{3}^{2}}$ and 
$\alpha=\cos ^{-1} \frac{K_{3}}{\sqrt{K_{2}^{2}+K_{3}^{2}}}$, then Eqs. ([12) and ([13]) become

$$
\begin{aligned}
& x(t)=-a \cos (\theta+\alpha) \\
& y(t)=\left(\frac{1}{\rho}\right) K_{1}+\left(\frac{a}{\rho}\right)\left[1+\left(\frac{1}{\rho}\right)\right] \sin (\theta+\alpha)
\end{aligned}
$$

It can be seen that the parameters $K_{1}, a$ and $\alpha$ correspond to the deviations from the center, size and initial phase, respectively. Figure $\square$ shows periodic orbits family corresponds to $\left(K_{1}, \alpha\right)=(0,1 / \sqrt{2})$ and $\left(K_{1}, \alpha\right)=(6,1 / \sqrt{2})$, respectively, drawn by varying $a$ and $\theta$ in Eqs. (155) and ([16). In Fig. Z, the corresponding values of $\boldsymbol{K}$ are also given. Note that the initial state on the periodic orbit depends on the initial true anomaly of the target $\theta$.

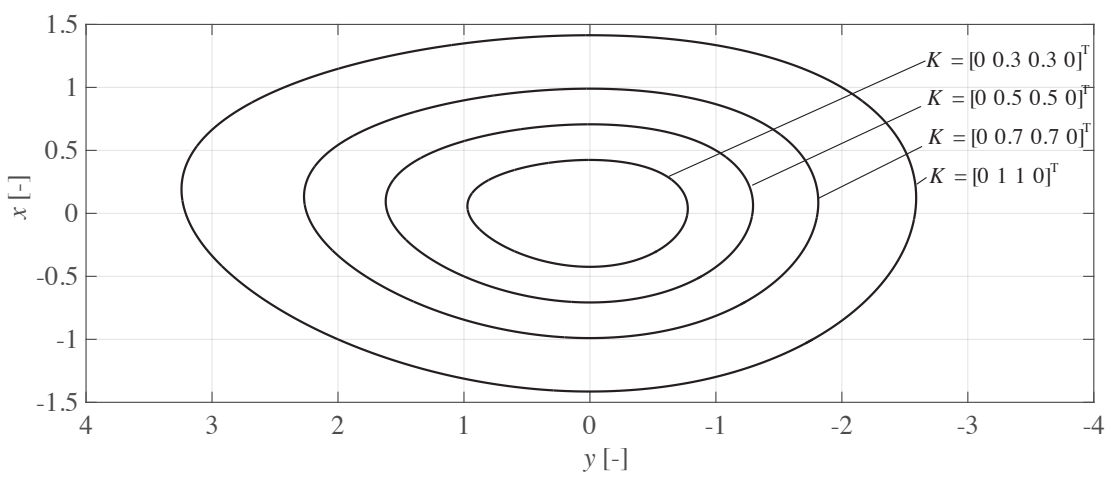

(a) $\left(K_{1}, \alpha\right)=(0,1 / \sqrt{2})$.

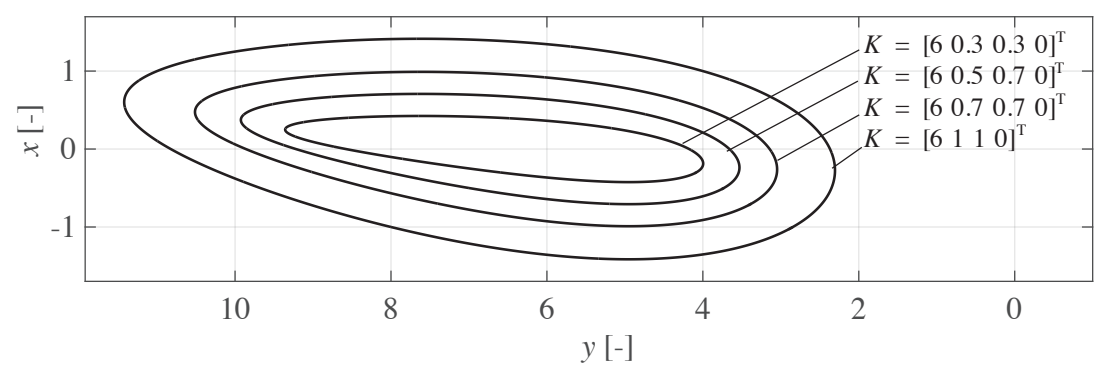

(b) $\left(K_{1}, \alpha=6,1 / \sqrt{2}\right)$.

Fig. 2 Periodic orbits family for $e=0.3$. 


\section{B. Optimal reconfiguration problem}

In this subsection, the optimal reconfiguration problem for formation flying is formulated and the attractive set of optimal control is introduced. For a given initial condition, the chaser is required to asymptotically track a final periodic relative orbit. The problem when the chaser is initially in a periodic relative orbit (an initial orbit) is called the reconstruction problem [16]. Let the state of the chaser be $\boldsymbol{X}_{1}$. Recall that the relative dynamics of the chaser are given by Eq. (四) so that

$$
\dot{\boldsymbol{X}}_{1}=A(t) \boldsymbol{X}_{1}+B \boldsymbol{u}, \quad \boldsymbol{X}_{1}\left(t_{0}\right)=\boldsymbol{X}_{10}
$$

where the initial condition $X_{10}$ satisfies the condition for periodic solutions $\left(K_{4}=0\right)$. To solve the reconfiguration problem, an error system is introduced:

$$
\dot{\boldsymbol{e}}=A(t) \boldsymbol{e}+B \boldsymbol{u}, \quad \boldsymbol{e}\left(t_{0}\right)=\boldsymbol{e}_{0}
$$

where $\boldsymbol{e}=\boldsymbol{X}_{1}-\boldsymbol{X}_{2}$, and $\boldsymbol{X}_{2}$ is the state of the final orbit

$$
\dot{\boldsymbol{X}}_{2}=A(t) \boldsymbol{X}_{2}, \quad \boldsymbol{X}_{2}\left(t_{0}\right)=\boldsymbol{X}_{20}
$$

where the initial condition $\boldsymbol{X}_{20}$ also satisfies the periodic condition. When $\boldsymbol{X}_{20}=0$, i.e. the final orbit is the origin of the coordinate, the problem is called a rendezvous problem, i.e. $\boldsymbol{e}=\boldsymbol{X}_{1}$. For simplicity, denote $\boldsymbol{X}$ to represent the state error for the reconfiguration problem or the state of the chaser for the rendezvous problem in the following. Consider an optimal control problem for Eq. (188) that minimizes the performance index $J$ given by

$$
J=\int_{t_{0}}^{\infty}\left(\boldsymbol{X}^{\mathrm{T}} Q \boldsymbol{X}+\boldsymbol{u}^{\mathrm{T}} R \boldsymbol{u}\right) d t
$$

where $Q \geq 0$ and $R>0$. Then, the optimal feedback control such that $X \rightarrow 0$ as $t \rightarrow \infty$ is given by

$$
\boldsymbol{u}^{*}=-R^{-1} B^{\mathrm{T}} M(t) \boldsymbol{X}
$$

where $M$ is a positive definite solution of the differential Riccati equation (DRE)

$$
-\dot{M}=A(t)^{\mathrm{T}} M+M A(t)+Q-M B R^{-1} B^{\mathrm{T}} M
$$


satisfying the final condition $\lim _{t_{f} \rightarrow \infty} M\left(t_{f}\right)=0$. If $\sqrt{Q}$ and $A$ are detectable, the DRE has a unique $T$-periodic stabilizing solution and its initial condition $M\left(t_{0}\right)$ is calculated recursively by

$$
-\dot{M}_{k}=A(t)^{\mathrm{T}} M_{k}+M_{k} A(t)+Q-M_{k} B R^{-1} B^{\mathrm{T}} M_{k}, \quad M_{k}(T)=M_{k-1}\left(t_{0}\right)
$$

with $M_{0}(T)=0$ [17]. In fact $M_{k}\left(t_{0}\right)$ monotonically converges to $M\left(t_{0}\right)$. Then the minimum value of the performance index $J^{*}$ is given by

$$
J^{*}=\boldsymbol{X}_{0}^{\mathrm{T}} M\left(t_{0}\right) \boldsymbol{X}_{0}
$$

Since $M\left(t_{0}\right) \geq 0$, the set $\left\{\boldsymbol{X}_{0} \in \mathbb{R}^{4} \mid \boldsymbol{X}_{0}^{\mathrm{T}} M\left(t_{0}\right) \boldsymbol{X}_{0} \leq C\right\}$ is an ellipsoidal set.

\section{Definition of Attractive Sets of Optimal Control}

For a given value of $J^{*}$, the states on the surface of Eq. (24) form an $n$-dimensional ellipsoid. Consider the set of all initial states inside the $n$-dimensional ellipsoid $J^{*}=C$

$$
\mathscr{A}(C)=\left\{\boldsymbol{X}_{0} \in \mathbb{R}^{n} \mid \boldsymbol{X}_{0}^{\mathrm{T}} M\left(t_{0}\right) \boldsymbol{X}_{0} \leq C\right\}
$$

The ellipsoidal set $\mathscr{A}(C)$ is called the attractive set of optimal control [14]. The optimal trajectory departing from the inside of the ellipsoid is guaranteed that the value of the performance index is less than $C$. It should be noted that the attractive set for a fixed final-state fixed finite-time optimal control problem is described by the controllability Grammian [14], while it is described by the positive definite solution of the Riccati equation for an infinite-time problem in Eq.(25]).

The important property of the attractive set is that the shape of ellipsoidal set is characterized by the eigenvalues and eigenvectors of the positive definite matrix $M\left(t_{0}\right)$. In fact, from the relation

$$
\lambda_{\min }\left(M\left(\left(t_{0}\right)\right)\left\|X_{0}\right\|^{2} \leq X_{0}^{T} M\left(t_{0}\right) X_{0} \leq \lambda_{\max }\left(M\left(\left(t_{0}\right)\right)\left\|X_{0}\right\|^{2}\right.\right.
$$

the largest distance of attractive set is defined by $\lambda_{\max }\left(M\left(t_{0}\right)\right)$ and the smallest one is defined by $\lambda_{\min }\left(M\left(t_{0}\right)\right)$. This property poses the problem of finding the optimal initial states over the set of possible initial states. 


\section{Attractive Sets for Formation Flying}

\section{A. Geometry of attractive sets for elliptic orbit}

For the TH equations, the attractive sets of optimal control are characterized by the solution to the DRE [Eq. (22)] and are given by

$$
\mathscr{A}\left(C, t_{0}\right)=\left\{\boldsymbol{X}_{0} \in \mathbb{R}^{4} \mid \boldsymbol{X}_{0}^{\mathrm{T}} M\left(t_{0}\right) \boldsymbol{X}_{0} \leq C\right\}
$$

From the definition [Eq. (27)], we can find the initial state to achieve $J^{*} \leq C$ at time $t_{0}$. Thus we call the attractive set [Eq. (27)] the instantaneous attractive sets in this paper. Since $M\left(t_{0}\right)$ depends on the initial true anomaly of the target $\theta_{0} \in[0,2 \pi]$, the shape of instantaneous attractive set [Eq. (27)] also depends on the true anomaly of the target. Figure 3$]$ illustrates the instantaneous attractive sets for various target's initial true anomaly $\theta_{0}$. It should be noted that the initial state of the chaser on the periodic orbit is uniquely determined by target's initial true anomaly and the instantaneous attractive sets vary with it. Therefore instantaneous attractive sets cannot be used to find the optimal initial position along a periodic orbit. This time-varying property of attractive sets makes it difficult to find the optimal initial state along a given initial periodic orbit geometrically.

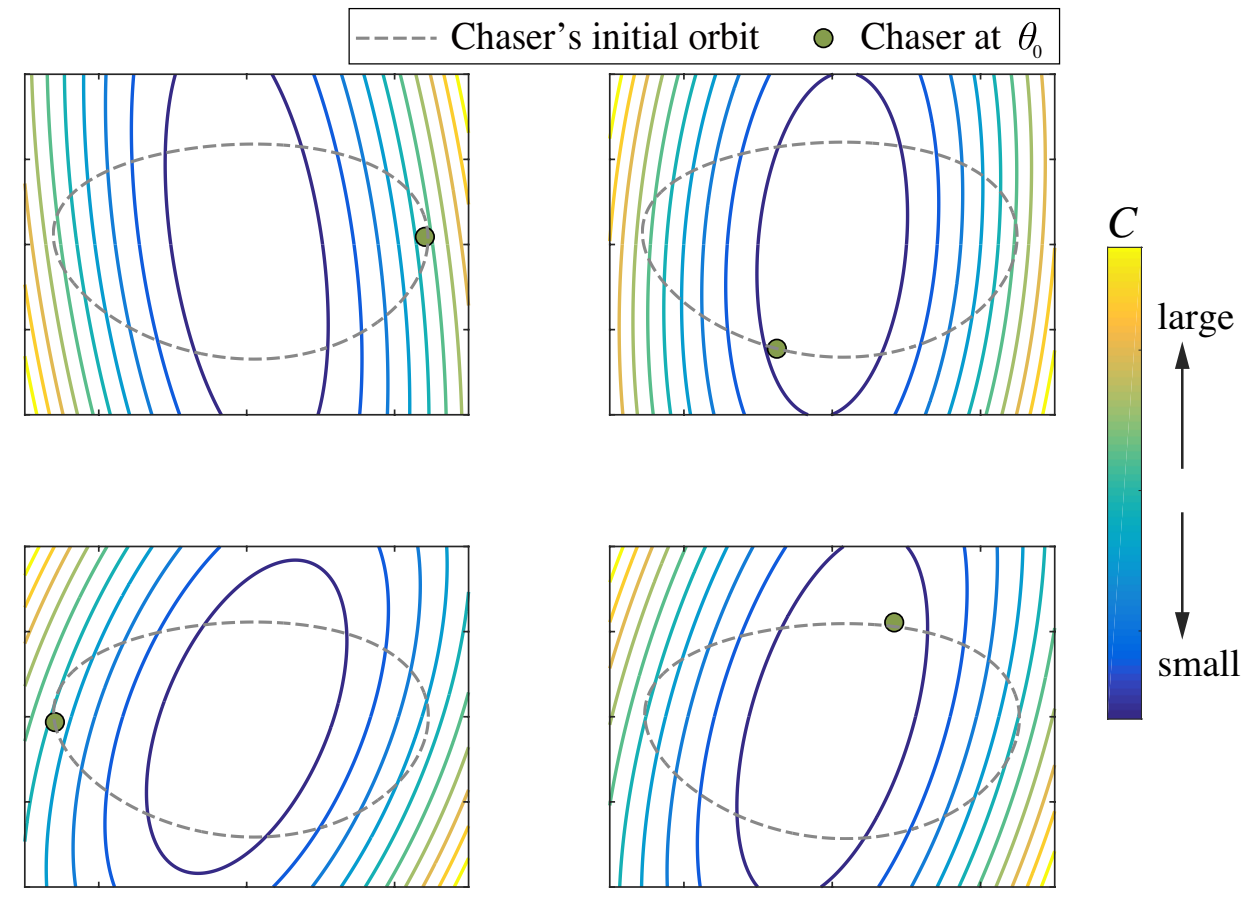

Fig. 3 Examples of instantaneous attractive sets: the shape of attractive sets and chaser's initial position depend on the target's initial true anomaly. 


\section{Attractive set associated with periodic orbits family of TH systems}

The chaser's initial orbit is assumed to be a periodic orbit of the TH equations and the attractive sets along periodic orbit family are depicted to find the optimal initial state on the periodic orbit. In the following, four-dimensional attractive sets are reduced to two-dimensional sets by restricting the initial state of the chaser to generate periodic orbit families as in the circular case. Assuming $K_{4}=0$ (periodic condition), we can see that all periodic orbits are parameterized by three parameters $\left(K_{1}, a, \alpha\right)$. Recall that the shape of periodic orbits are determined by $K_{1}$ and $\alpha$, therefore a family of periodic orbits for specific $K_{1}$ and $\alpha$ denoted by $\Gamma\left(K_{1}, \alpha\right)$ is considered. Then all initial state of $\Gamma\left(K_{1}, \alpha\right)$ is parameterized by the size parameter $a>0$ and the initial true anomaly $0 \leq \theta_{0} \leq 2 \pi$ so that the corresponding initial position $\left(x_{0}, y_{0}\right)$ of the chaser is uniquely computed from Eqs. (II5) and (I6)). Corresponding initial velocity $\left(\dot{x}_{0}, \dot{y}_{0}\right)$ is also uniquely determined on $\Gamma\left(K_{1}, \alpha\right)$ from Eqs. (II5) and ([16). Then the attractive sets can be drawn in two-dimensional plane of $\left(x_{0}, y_{0}\right)$ as in the circular case. Thus, the projection of attractive sets onto periodic orbits family $\Gamma\left(K_{1}, \alpha\right)$ is defined as

$$
\mathscr{A}\left(C, \Gamma\left(K_{1}, \alpha\right)\right)=\left\{\left(x_{0}, y_{0}\right) \in \mathbb{R}^{2} \mid \boldsymbol{X}_{0}^{\mathrm{T}} M\left(\theta_{0}\right) \boldsymbol{X}_{0} \leq C, \boldsymbol{X}_{0} \in \Gamma\left(K_{1}, \alpha\right)\right\}
$$

Now the procedure to draw the attractive sets [Eq. ([28)] is presented below. The parameter $K_{1}$ corresponds to a translation of $y$, and hence $K_{1}=0$ is assumed for simplicity. The main observation here is that the shape of attractive sets depends on $K_{1}$ and $\alpha$, and it is independent of the size parameter $a$. For a given value of $C$, the procedure to draw the attractive set $\mathscr{A}\left(C, \Gamma\left(K_{1}, \alpha\right)\right)$ is as follows:

step 1 Let $\theta\left(t_{0}\right)=\theta_{0}=0$ (the target is initially at perigee) and $Q(0)$ be the chaser's initial position (see the green circle in Fig. 4(a)). Draw an instantaneous attractive set of unit size $\boldsymbol{x}_{0}^{\mathrm{T}} M(0) \boldsymbol{x}_{0}=1$ (see the red solid line in Fig. 4(a)).

step 2 Draw a straight line $\overline{O Q(0)}$ from the origin to the position of the chaser (the dotted black line in Fig. 4(a)). Let $P(0)$ be the intersecting point with the segment $\overline{O Q(0)}$ and the unit instantaneous attractive set (see the blue circle in Fig. 4(a)).

step 3 The attractive set at $\theta_{0}=\theta$ (the target is initially at $\theta$ ) can be drawn in a similar manner. Draw the unit instantaneous attractive set $\boldsymbol{x}_{0}^{\mathrm{T}} M(\theta) \boldsymbol{x}_{0}=1$ (the red solid line in Fig. 4(b)). Then draw a straight line $\overline{O Q(\theta)}$ from the origin to the position of the chaser at $\theta$, and let $P(\theta)$ be the intersecting point with $\overline{O Q(\theta)}$ and the unit instantaneous attractive set (the blue circle in Fig. 4(b)). Repeat step 3 for $\theta_{0}$ from 0 to $2 \pi$.

step 4 The attractive set along the initial orbit of the chaser is generated by connecting $P\left(\theta_{0}\right)\left(\theta_{0}\right.$ from 0 to $\left.2 \pi\right)$ (the solid blue line in Fig. 4(c)) .

step 5 Finally the attractive set of a desired value $C$ is obtained by multiplying a scaling factor $C$ (see the solid red line Fig. 4(d)]). 
From the geometry of attractive sets, the largest attractive set inscribed in the initial periodic orbit and their tangent point are the minimum cost and the optimal initial position along the periodic orbit. Therefore, the optimal initial state on the initial periodic orbit can be sought as the point of contact between the maximum attractive set inscribed in the initial periodic orbit and the initial periodic orbit.

\section{B. Geometry of attractive sets for elliptic orbit: general case}

In the previous subsection, the translation of periodic orbits from the center is assumed to be zero, i.e. $K_{1}=0$. In the case of $K_{1} \neq 0$, periodic orbit family and attractive sets no more have their common center. Instead, the center of periodic orbit at each instant is given by $(x, y)=\left(0, \frac{K_{1}}{1+e \cos \theta}\right)$ from Eq. (114). Then, in step 2 of the previous subsection, it is necessary to draw a straight line from the center of the periodic orbit at each instant. Therefore, the procedure is modified as follows:

step 1 Let $\theta\left(t_{0}\right)=\theta_{0}=0$ (the target is initially at perigee) and $Q(0)$ be the chaser's initial position (the green circle in Fig. 5(a)). Then draw an instantaneous attractive set of unit size $\boldsymbol{x}_{0}^{\mathrm{T}} M(0) \boldsymbol{x}_{0}=1$ (the dotted blue line in Fig. 5(a)).

step 2 Draw a straight line $\overline{O(0) Q(0)}$ from the center at $\theta_{0}=0$ denoted by $O(0)$. Let $P(0)$ be the intersecting

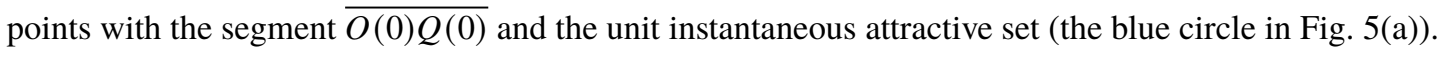

step 3 The attractive set at $\theta_{0}=\theta$ (the target is initially at $\theta$ ) can be determined in a similar manner. Draw a unit instantaneous attractive set $\boldsymbol{x}_{0}^{\mathrm{T}} M(\theta) \boldsymbol{x}_{0}=1$. Then draw a straight line $\overline{O(\theta) Q(\theta)}$ from the center at $\theta$ denoted by $O(\theta)$, and let $P(\theta)$ be the intersecting point with $\overline{O(\theta) Q(\theta)}$ and the unit instantaneous attractive set. Repeat step 3 for $\theta_{0}$ from 0 to $2 \pi$ (the dotted blue line in Fig. 5(b)) .

step 4 The attractive set along the initial orbit of the chaser is generated by $P\left(\theta_{0}\right)$ for $\theta_{0}$ from 0 to $2 \pi$ (the solid blue line in Fig. $5(\mathrm{c}))$.

step 5 Finally the attractive set of a desired value $C$ is obtained by multiplying a scaling factor $C$ (the solid red line Fig. (d(d))

Even in the general case, the largest attractive set inscribed in the initial periodic orbit and their tangent point are the minimum cost and the optimal initial position along the periodic orbit. Therefore, the optimal initial state on the initial periodic orbit can be also sought as the point of contact.

\section{Numerical Simulations}

In the numerical simulation, the nondimensional equations of relative motion are used: distances are nondimensionalized utilizing the target's radius and time is also nondimensionalized by the mean motion of target's orbit. The 


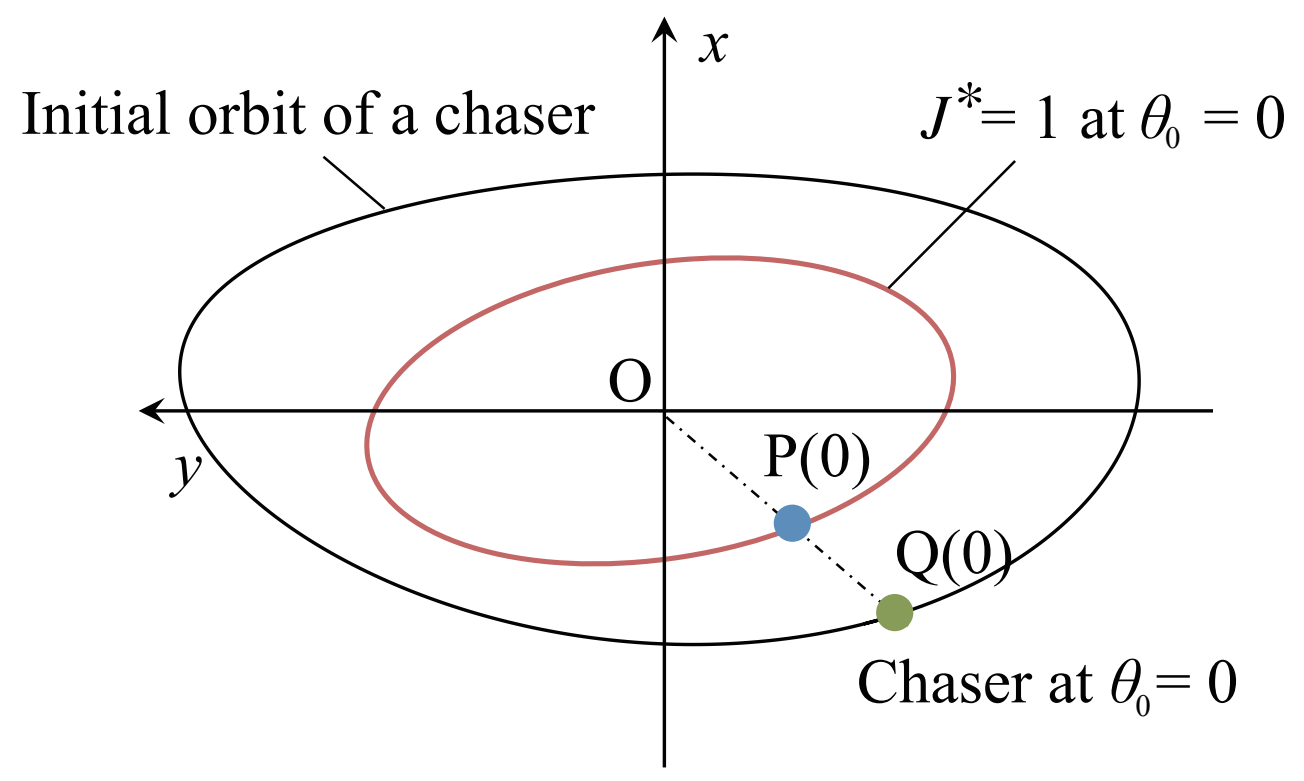

(a) step 1 and 2

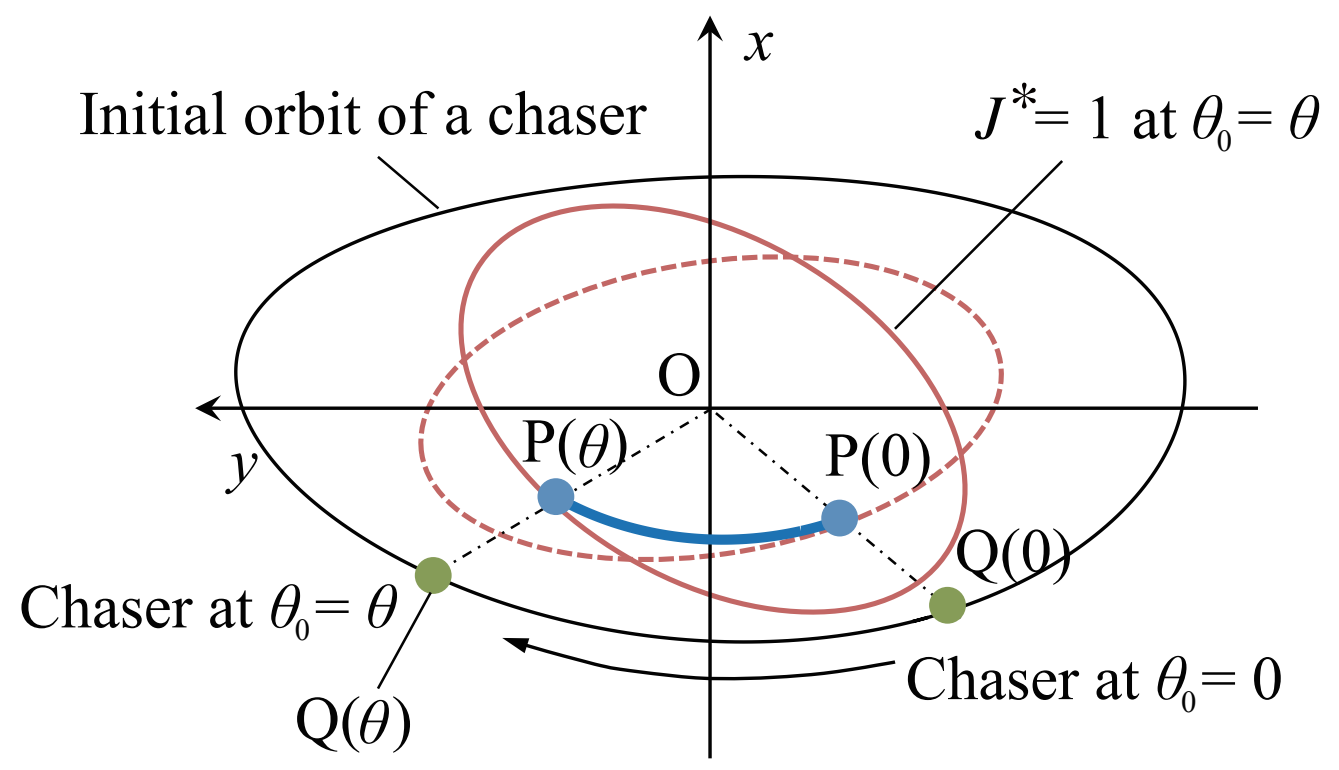

(b) step 3 


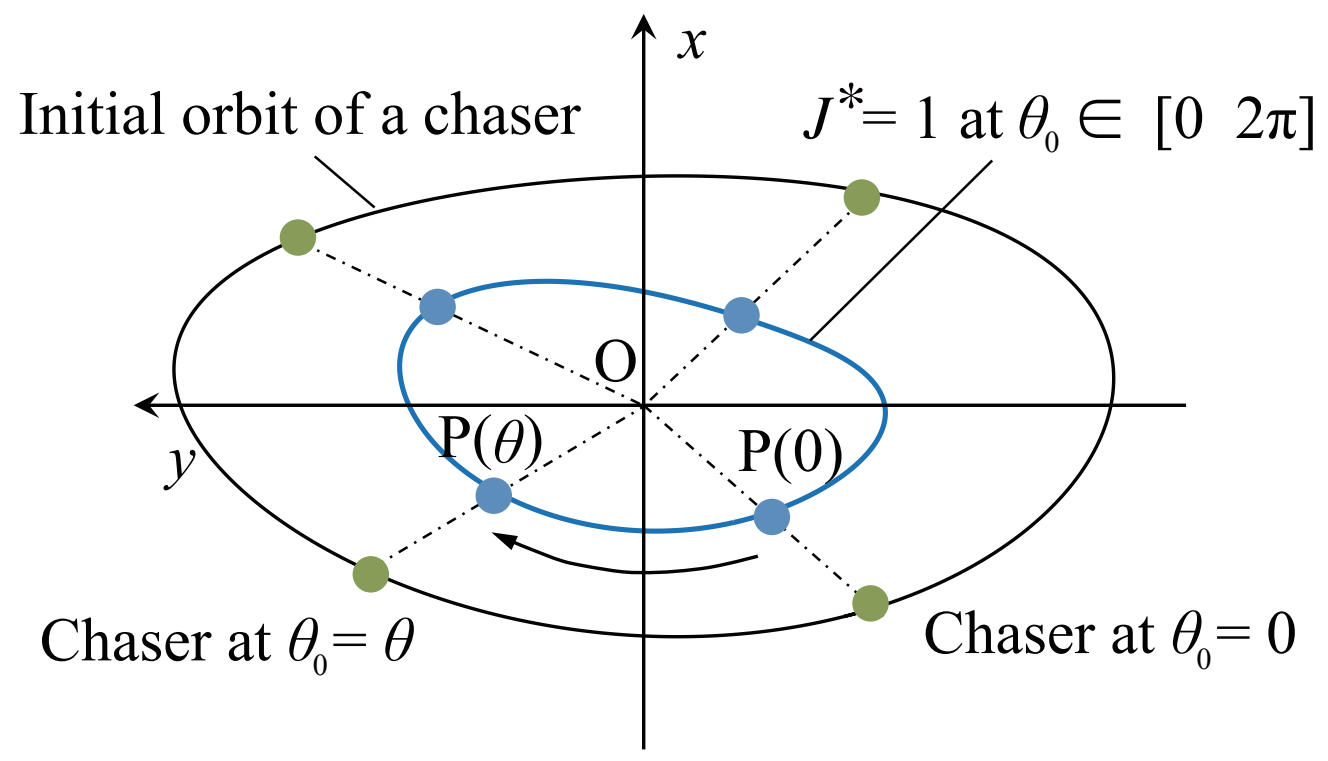

(c) step 4

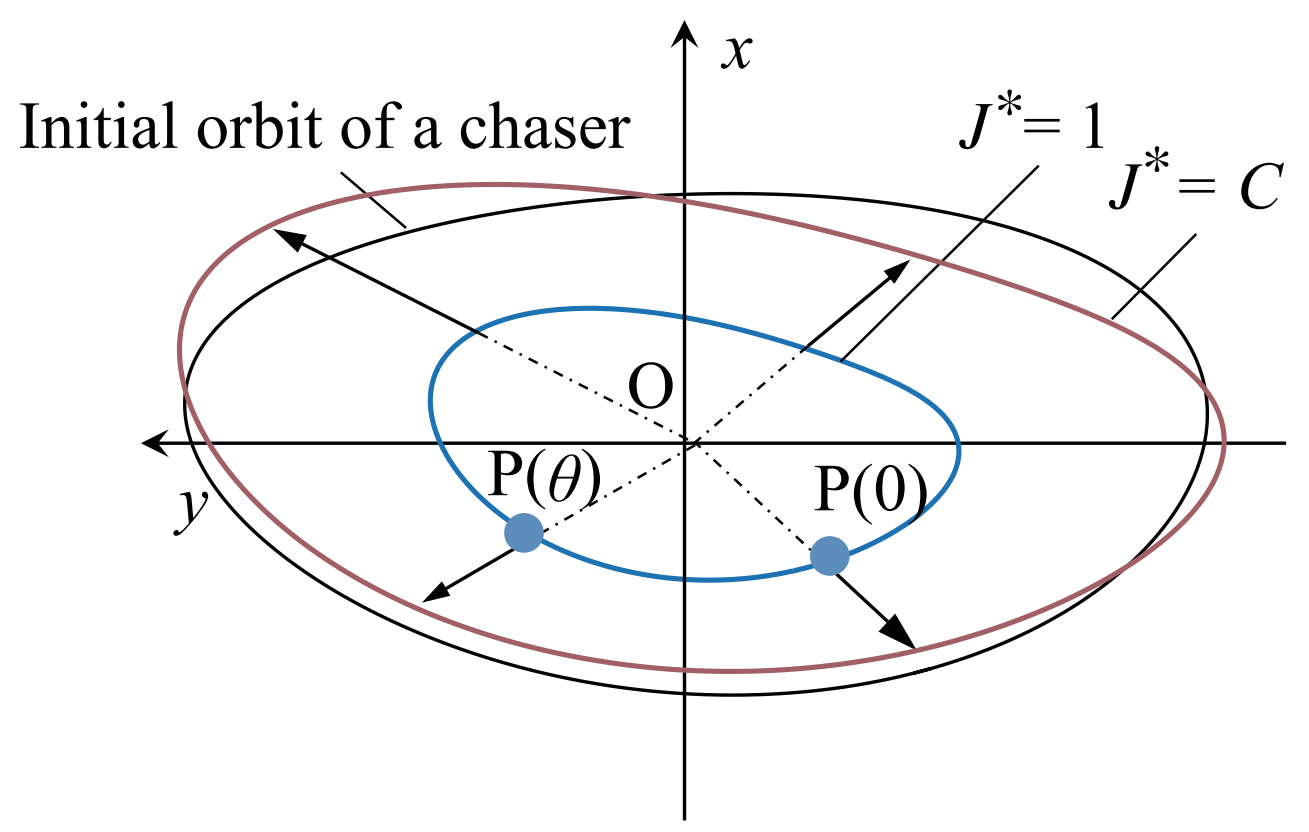

(d) step 5

Fig. 4 How to construct the attractive sets along elliptic orbit. 


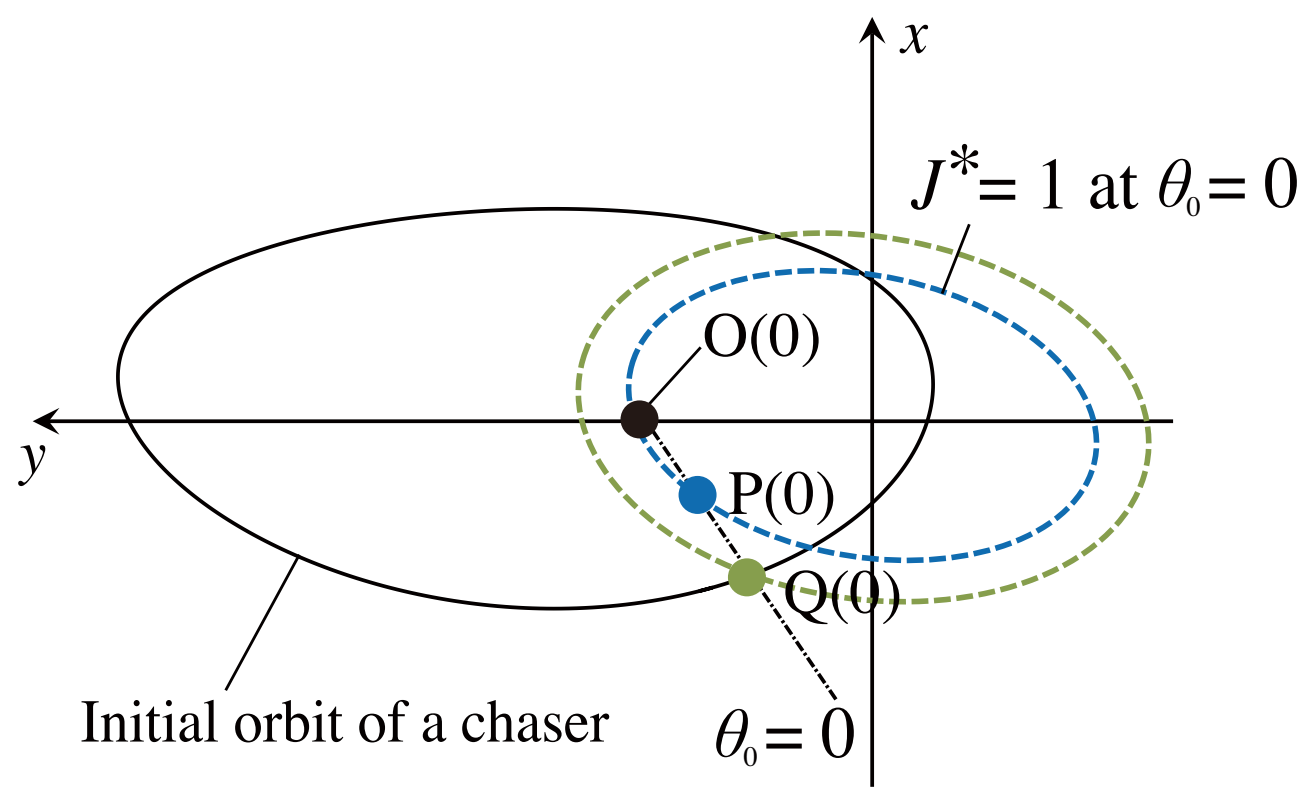

(a) step 1 and 2

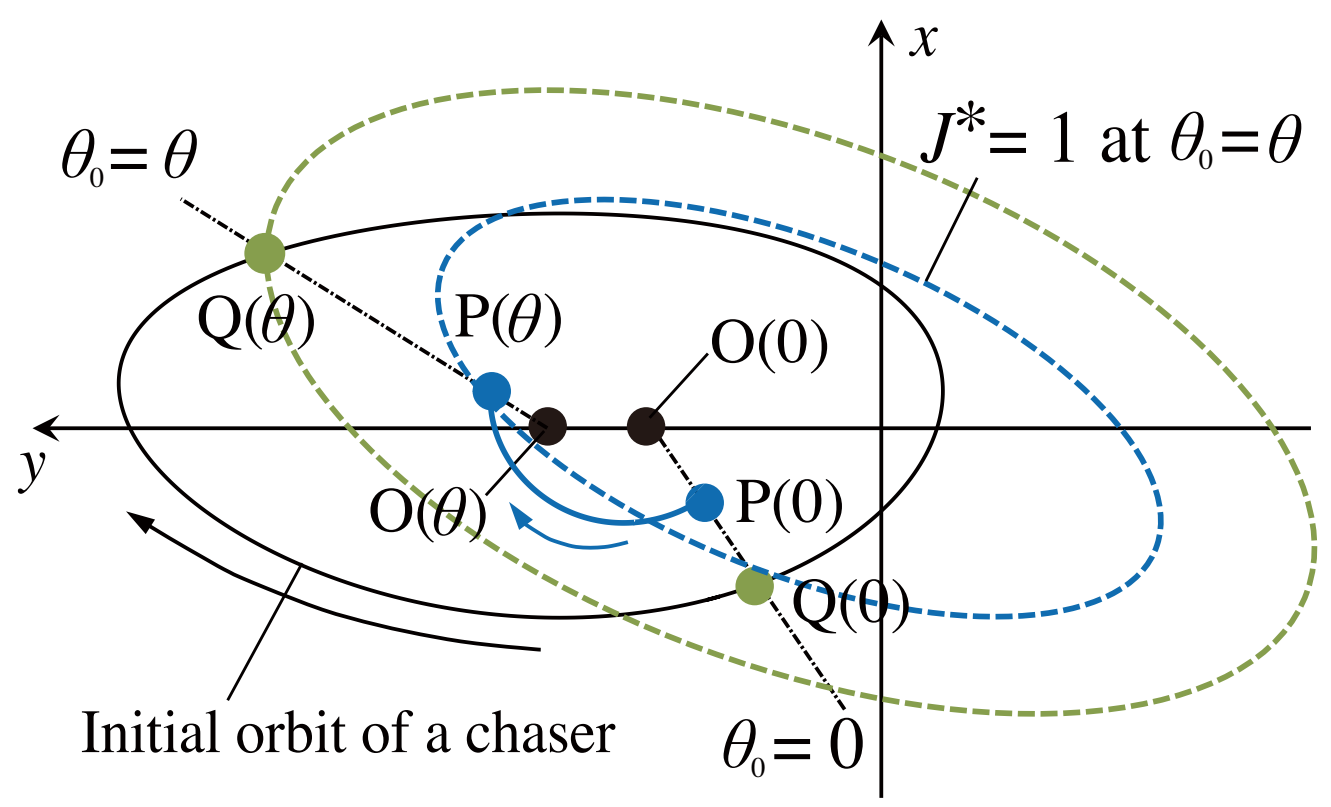

(b) step 3 


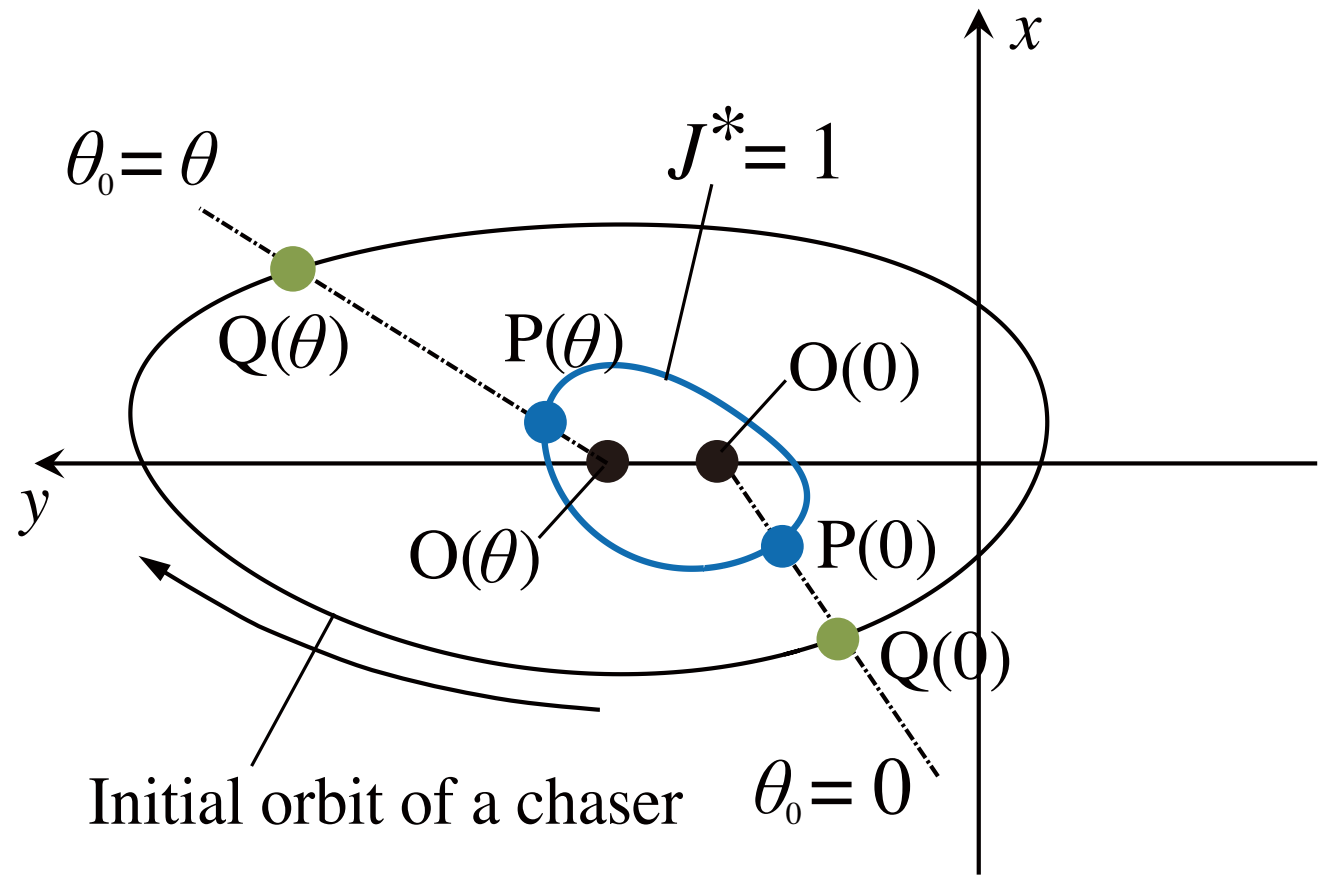

(c) step 4

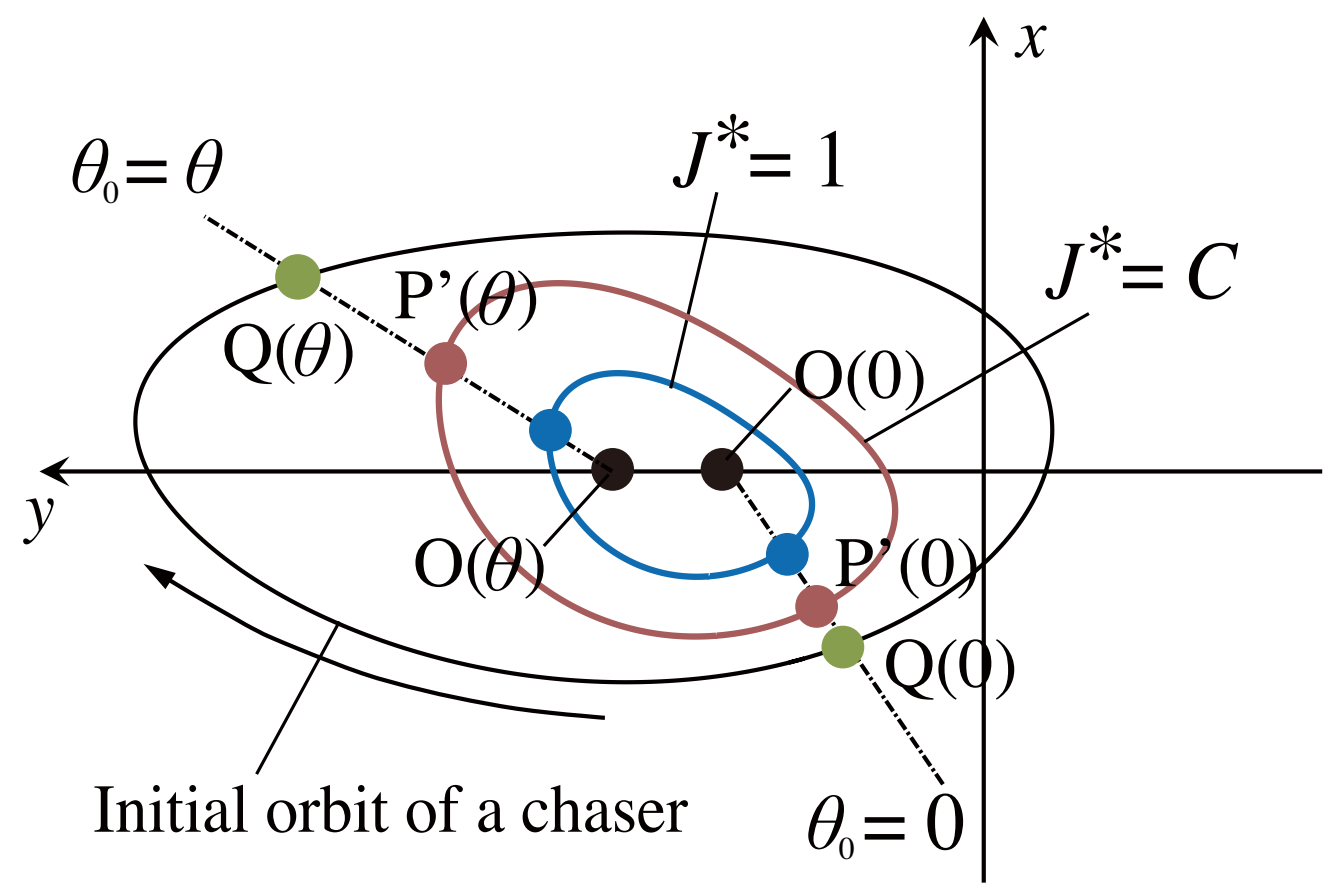

(d) step 5

Fig. 5 How to construct the attractive sets along elliptic orbit $\left(K_{1} \neq 0\right)$. 
feedback control is designed to achieve rendezvous with a target at the origin to minimize the performance index

$$
J=\int_{0}^{\infty}\left(\boldsymbol{X}^{\mathrm{T}} Q \boldsymbol{X}+\boldsymbol{u}^{\mathrm{T}} R \boldsymbol{u}\right) d t
$$

In the following, the weight matrices in Eq. (2Q) are assumed to be $Q=10^{q} \boldsymbol{I}, R=\boldsymbol{I}$ and the $L_{2}$-norm of the control is defined as

$$
\|\boldsymbol{u}\|_{2}=\left(\int_{t_{0}}^{t_{f}}|\boldsymbol{u}|^{2} d t\right)^{\frac{1}{2}}
$$

where the setting time $t_{f}$ is the first time after which inequality $|X(t)| \leq 1.0 \times 10^{-2}$ is satisfied.

\section{A. Optimal Rendezvous Problem for the TH Equations}

In this subsection, attractive sets for optimal rendezvous problem along an elliptic orbit is presented and the geometries of the attractive sets are investigated based on the proposed procedure. For convenience, the attractive sets presented in Section III can be plotted utilizing the MATLAB ${ }^{\circledR}$ function. First the time-periodic solution $M(\theta(t))$ of the differential Riccati equation [Eq. ([2])] is obtained by using Eq. ([23). Then for each $\theta$, the instantaneous attractive set of unit size $\boldsymbol{x}_{0}^{\mathrm{T}} M(\theta) \boldsymbol{x}_{0}=1$ is plotted by using contour function of MATLAB ${ }^{\circledR}$. A rough estimate for the largest attractive set inscribed in the initial orbit is graphically found by varying the value of scaling factor $C$.

\section{Optimal trajectory design using attractive sets}

For the TH equations, the optimal control is given by the time-periodic solution to the differential Riccati equation [Eq. (22)], while it is given by the solution to the algebraic Riccati equation for the HCW equations. The attractive sets of optimal reconfiguration control problem is implemented by its periodic solution obtained by Eq. ([23]). Assume that the target's orbit is elliptic with eccentricity of 0.3 and the chaser is initially on a periodic orbit corresponding to $\boldsymbol{K}=\left[\begin{array}{llll}0 & 1 & 2 & 0\end{array}\right]^{\mathrm{T}}$ which corresponds to $\left(K_{1}, \alpha\right)=(0, \sqrt{5})$. Figure 6 shows the contour of the attractive sets [Eq. (27)] for different weight parameters obtained by the proposed procedure. Figure $\square$ shows the optimal trajectories of the chaser for $\theta_{0}=0$, i.e. the target is initially at perigee. In Fig. Z, the black lines represent the chaser's initial periodic orbit, the blue circles represent the chaser's initial state, and the red lines represent the controlled trajectories of the chaser. In our geometric approach, the optimal initial states are obtained as the tangent point between the largest attractive set inscribed in the chaser's initial periodic orbit and the initial periodic orbit. Figure $\mathbb{8}$ shows the optimal trajectories of the chaser for the optimal initial state along the initial periodic orbit obtained by the proposed procedure. On the other hand, since the chaser's initial periodic orbit is 1-dimensional manifold on $\theta_{0} \in[0,2 \pi]$, the problem to 
Table 1 Performance indices: elliptic case $\left(K=\left[\begin{array}{lll}0 & 1 & 2\end{array}\right]^{\mathrm{T}}\right)$.

\begin{tabular}{ccccccc}
\hline \hline$q$ & \multicolumn{2}{c}{$J^{*}[-]$} & \multicolumn{2}{c}{$t_{f}[-]$} & \multicolumn{2}{c}{$L_{2}$-norm [-] } \\
& $\theta_{0}=0$ & $\theta_{0}=\theta_{0}^{*}$ & $\theta_{0}=0$ & $\theta_{0}=\theta_{0}^{*}$ & $\theta_{0}=0$ & $\theta_{0}=\theta_{0}^{*}$ \\
\hline 3 & 8538 & 5499 & 5.338 & 9.295 & 27.98 & 17.17 \\
0 & 47.63 & 18.25 & 7.645 & 10.22 & 3.729 & 2.333 \\
-5 & 0.00253 & 0.00252 & 628.9 & 629.9 & 0.1502 & 0.1501 \\
\hline \hline
\end{tabular}

find the optimal initial true anomaly $\theta_{0}^{*}$ on a initial periodic orbit is stated as

$$
\begin{aligned}
& \text { Find } \theta_{0}^{*}=\underset{0 \leq \theta_{0} \leq 2 \pi}{\arg \min } \boldsymbol{x}_{0}\left(\theta_{0}\right)^{\mathrm{T}} X\left(\theta_{0}\right) \boldsymbol{x}_{0}\left(\theta_{0}\right) \\
& \text { subject to } \frac{x_{0}^{2}\left(\theta_{0}\right)}{k_{2}^{2}+k_{3}^{2}}+\frac{\left[y_{0}\left(\theta_{0}\right)-\left(\frac{k_{1}}{\rho\left(\theta_{0}\right)}\right)\right]^{2}}{\left[1+\left(\frac{1}{\rho\left(\theta_{0}\right)}\right)\right]^{2}\left(k_{2}^{2}+k_{3}^{2}\right)}=1, \quad 0 \leq \theta_{0} \leq 2 \pi
\end{aligned}
$$

The optimal initial position obtained by the proposed method were validated by the solution obtained by a numerical

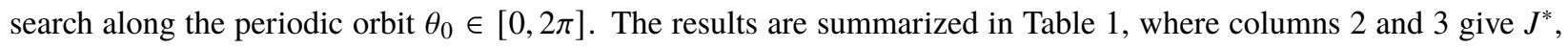
columns 4 and 5 the flight time and columns 6 and 7 the $L_{2}$-norm. The performance index $J^{*}$ is minimized when the optimal initial state is used. It can be seen that the controlled trajectory starting from the optimal initial position reduces the cost $J^{*}$ but takes a slightly longer flight time for the same $q$. Moreover, as $q$ decreases, the $L_{2}$-norm of control decreases while $t_{f}$ increases.

\section{Discussion on a shape of attractive sets}

In order to further understand the relations between the weight matrices and the obtained optimal trajectories, the changes of the shape of the attractive sets is investigated for some weight parameters $q$. In Fig. G(a), the shape of the contour is like a circle when the weight on state is relatively large $(q=3)$. This result implies that the optimal trajectory tends to take a shortest path to the origin for high energy trajectories. Indeed, the distance to the origin is the shortest on $(x, y)=(2,0)$ on the periodic orbit. As the weight on control input becomes relatively large $(q=-5)$, the shape of attractive sets have the same shape with that of the initial periodic orbit. From the geometry of the sets, the minimum values of the performance index occur at the tangent points between the periodic orbit and the attractive sets, which overlaps the contour of $J^{*}$. These results suggest that the asymptotic behavior of the attractive sets to reflect the free motion and to become less sensitive to the initial state as presented in [15] for circular case holds for the TH equations.

\section{Attractive sets for general case}

A general case with $\boldsymbol{K}=\left[\begin{array}{lllll}6 & 1 & 1 & 0\end{array}\right]^{\mathrm{T}}$ is considered as an example. The attractive sets drawn by the procedure presented in Sec. II.B are shown in Fig. Q. From Fig. Q, the attractive sets converge to the shape of the periodic 


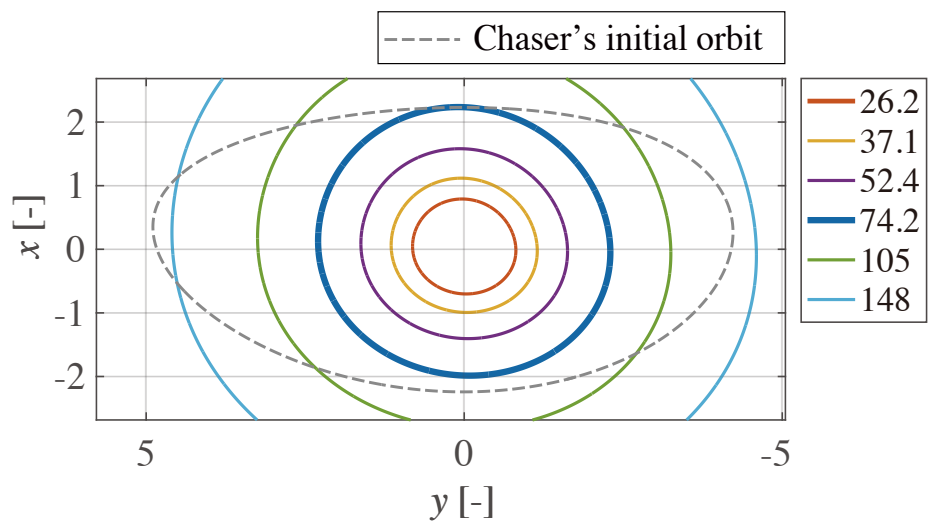

(a) $q=3$.

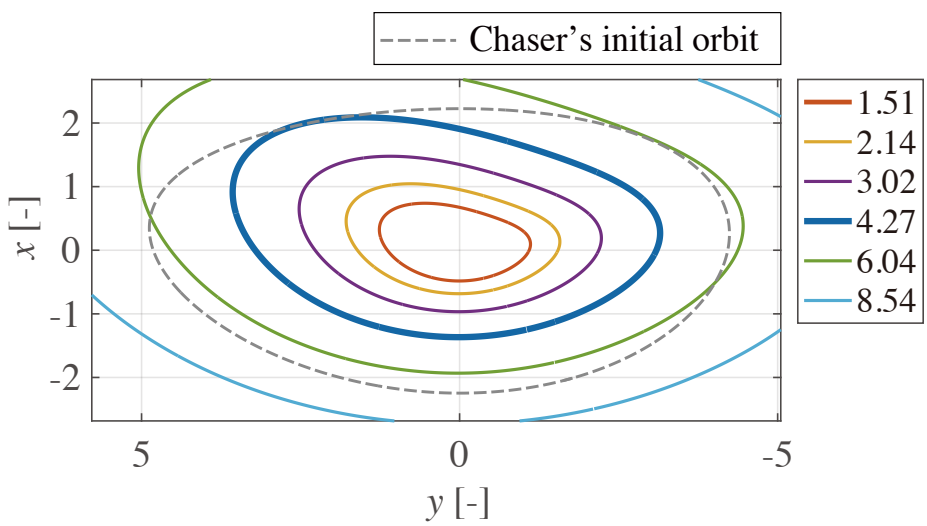

(b) $q=0$.

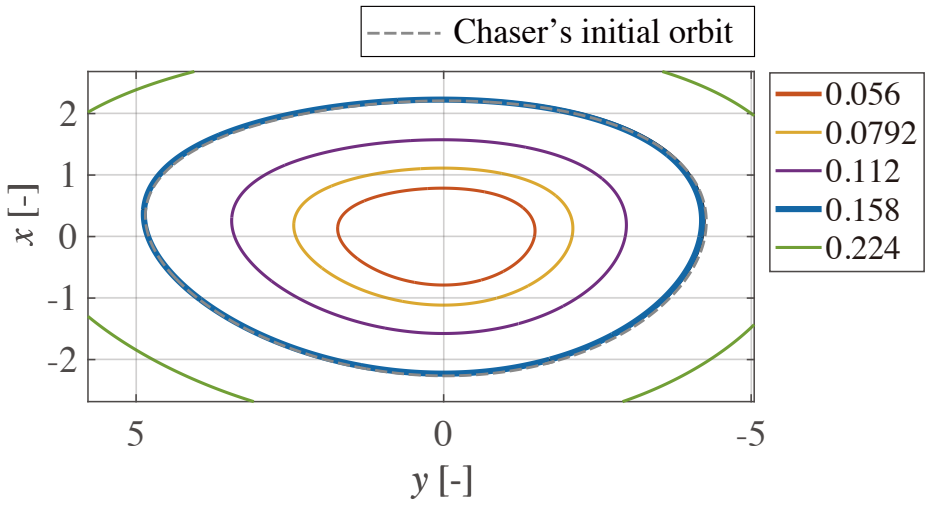

(c) $q=-5$.

Fig. 6 The contour of the attractive set for $q=3,0,-5$. 


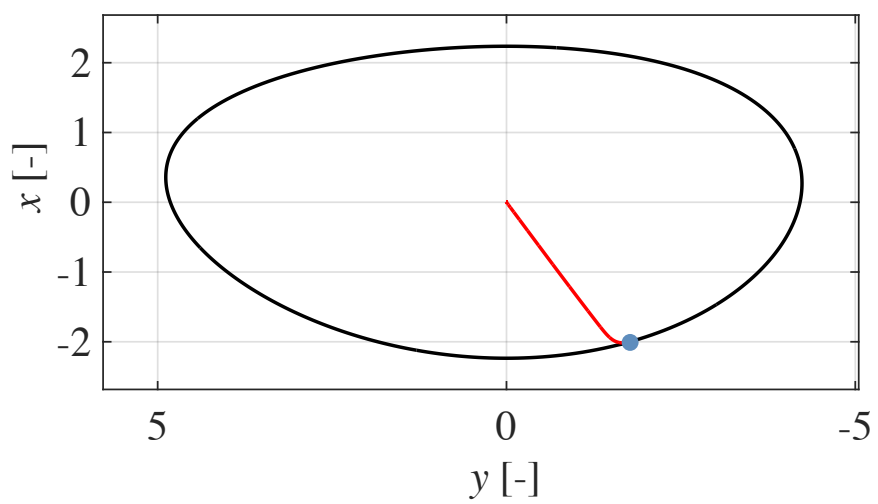

(a) Optimal trajectory for $q=3$ : $\theta_{0}=0$.

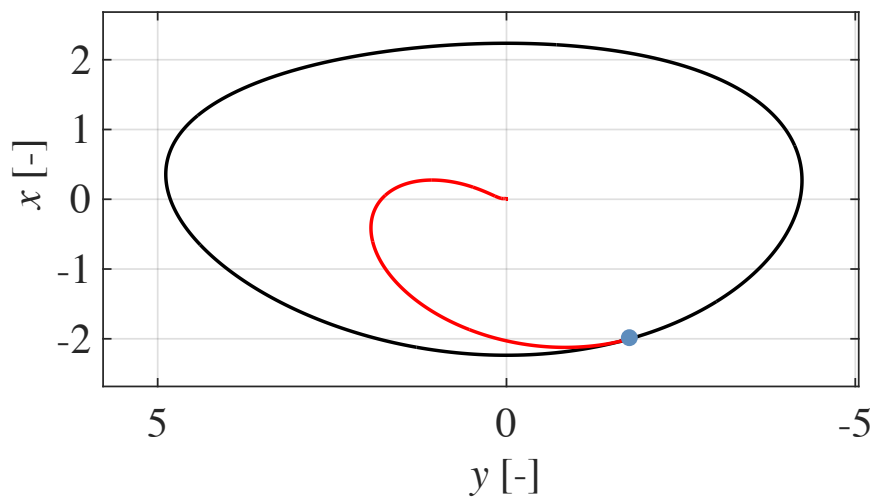

(b) Optimal trajectory for $q=0$ : $\theta_{0}=0$.

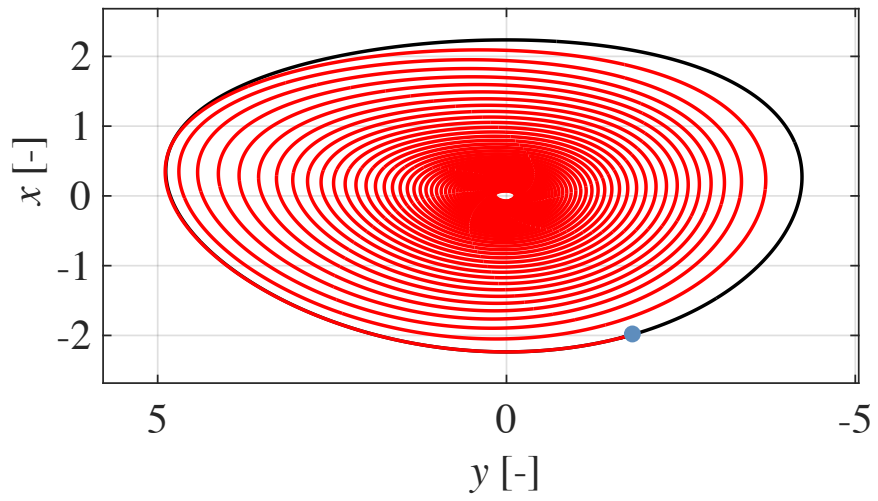

(c) Optimal trajectory for $q=-5: \theta_{0}=0$.

Fig. 7 The optimal trajectories for $\theta_{0}=0$. Target's initial true anomaly is fixed to perigee. 


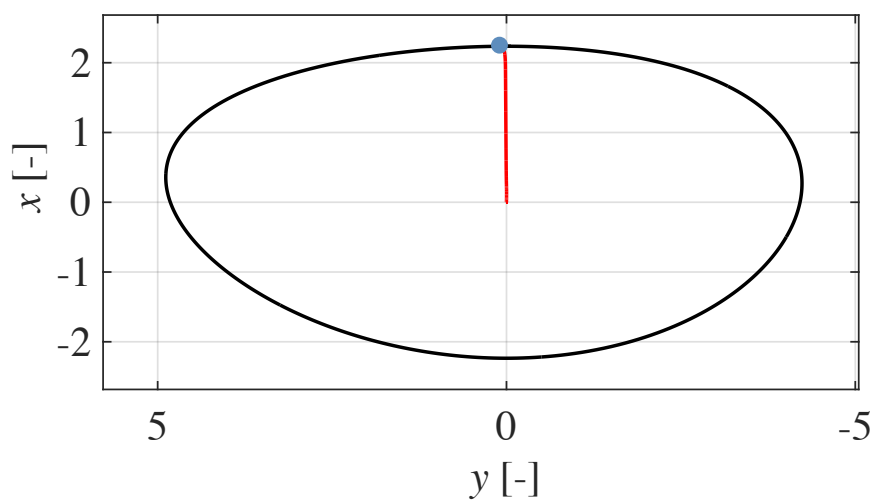

(a) Optimal trajectory for $q=3$ : optimal initial state.

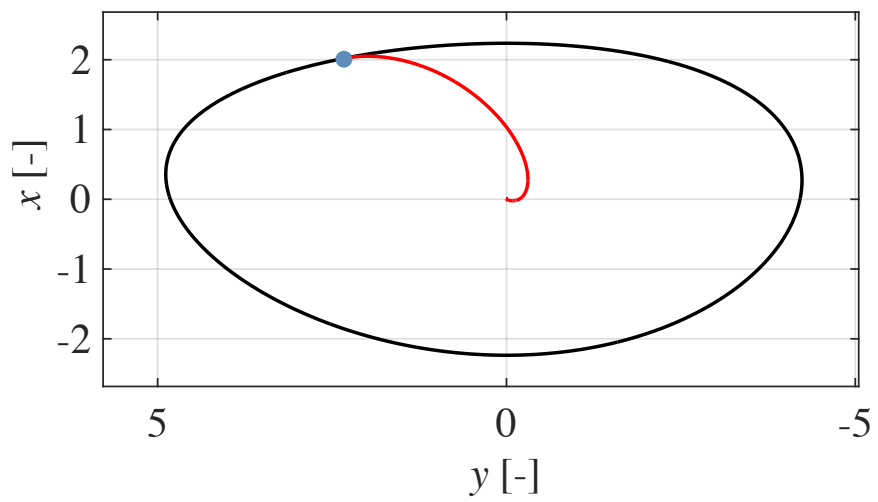

(b) Optimal trajectory for $q=0$ : optimal initial state.

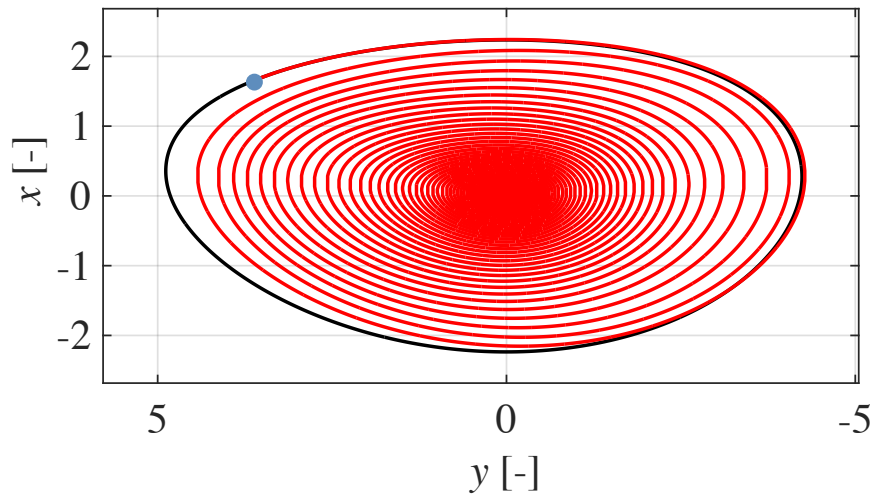

(c) Optimal trajectory for $q=-5$ : optimal initial state.

Fig. 8 The optimal trajectories for the optimal initial state. Target's initial true anomaly is optimized. 
Table 2 Performance indices: elliptic case $\left(K=\left[\begin{array}{lll}6 & 1 & 1\end{array}\right]^{\mathrm{T}}\right)$.

\begin{tabular}{ccccccc}
\hline \hline$q$ & \multicolumn{2}{c}{$J^{*}[-]$} & \multicolumn{2}{c}{$t_{f}[-]$} & \multicolumn{2}{c}{$L_{2}$-norm $[-]$} \\
& $\theta_{0}=0$ & $\theta_{0}=\theta_{0}^{*}$ & $\theta_{0}=0$ & $\theta_{0}=\theta_{0}^{*}$ & $\theta_{0}=0$ & $\theta_{0}=\theta_{0}^{*}$ \\
\hline 3 & 10902 & 5806 & 5.703 & 11.37 & 32.82 & 16.12 \\
0 & 92.20 & 22.18 & 8.442 & 12.74 & 5.936 & 2.712 \\
-4 & 0.1971 & 0.1675 & 181.2 & 187.3 & 0.2848 & 0.2862 \\
\hline \hline
\end{tabular}

orbit even in the case where $K_{1}$ is a nonzero value. Figure [0 shows the optimal trajectories of the chaser for $\theta_{0}=0$. Figure $\mathbb{}$ s] shows the optimal trajectory departing from the optimal initial state obtained by the proposed geometric approach. The optimal initial position obtained by the proposed method were also validated by the solution obtained by a numerical search along the periodic orbit $\theta_{0} \in[0,2 \pi]$. The results are summarized in Table $\square$. The optimal initial position reduces the cost $J^{*}$ but takes a longer flight time for the same $q$ as in the previous case. In the optimal controlled trajectory, it is observed that the trajectories starting from the optimal initial states exploit the natural periodic motion more effectively. 


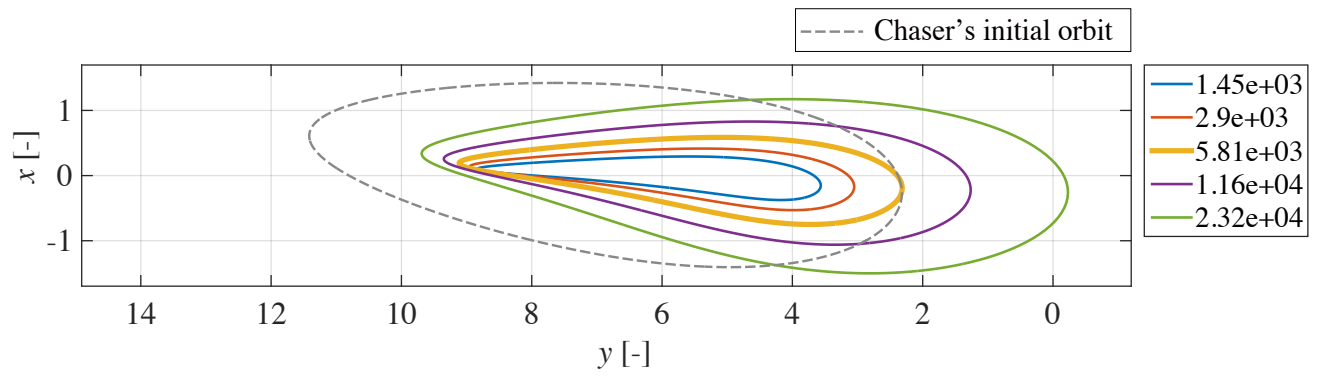

(a) $q=3$.

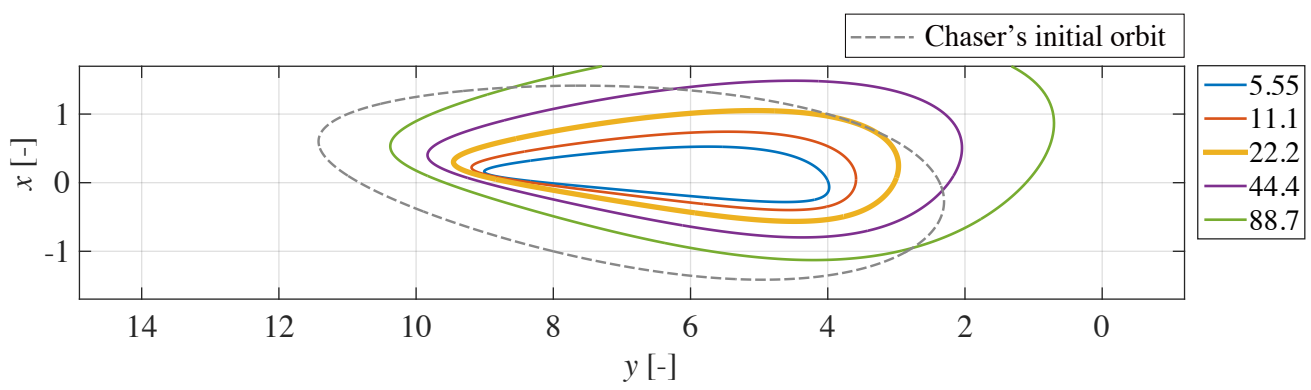

(b) $q=0$.

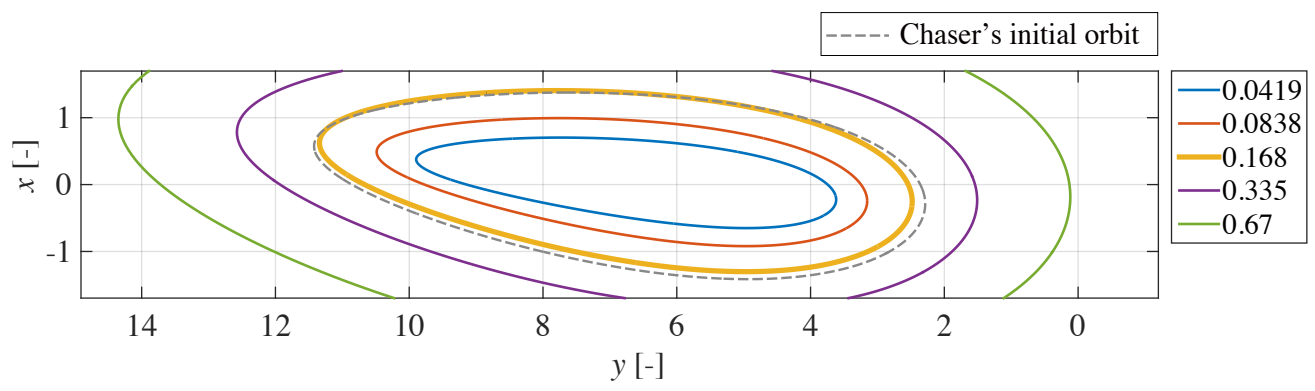

(c) $q=-4$.

Fig. 9 The contour of the attractive set for $q=3,0,-4$. 


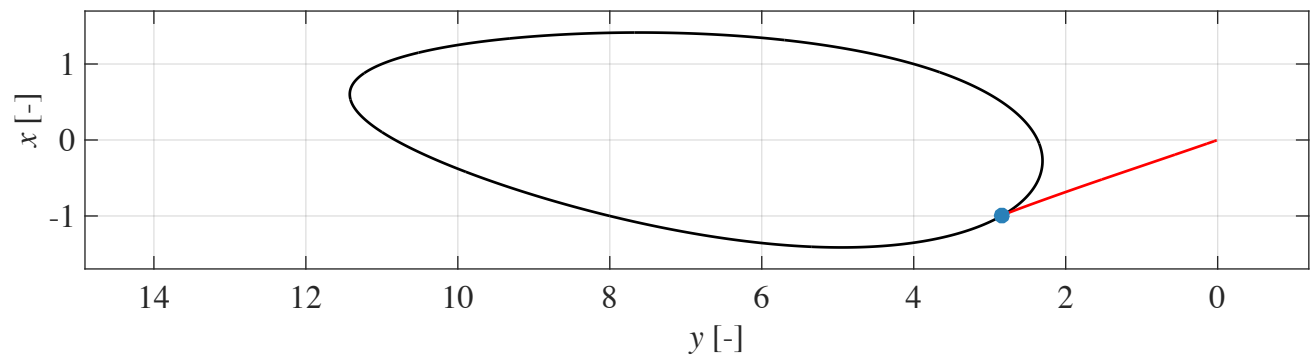

(a) $q=3$.

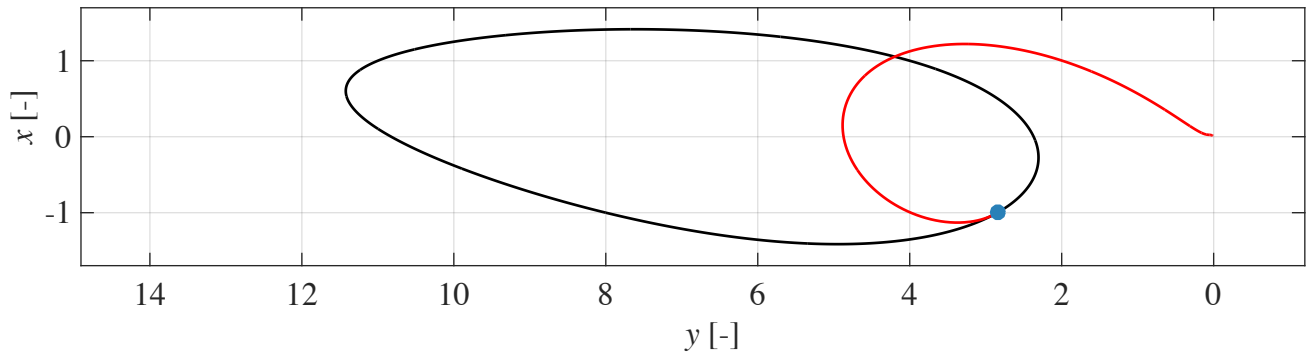

(b) $q=0$.

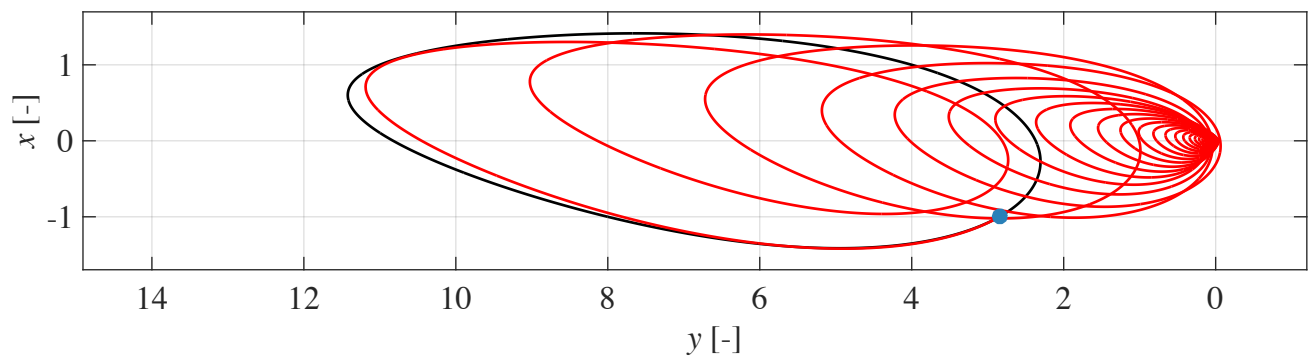

(c) $q=-4$.

Fig. 10 The optimal controlled trajectories corresponding to $\theta_{0}=0$ for $q=3,0,-4$. 


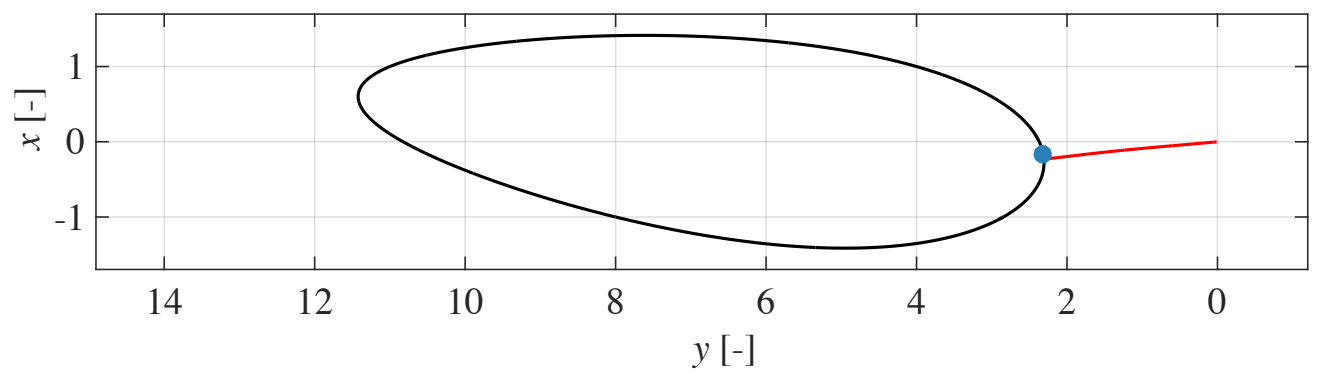

(a) $q=3$.

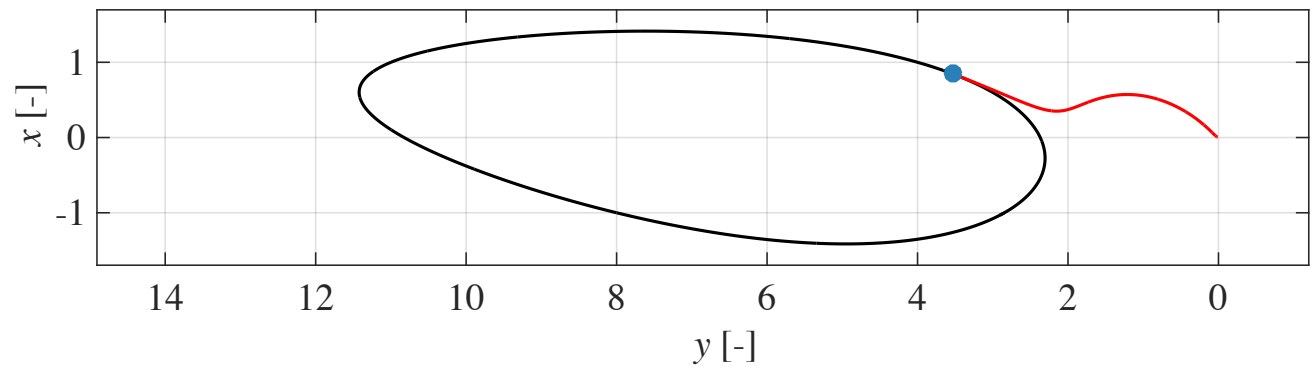

(b) $q=0$.

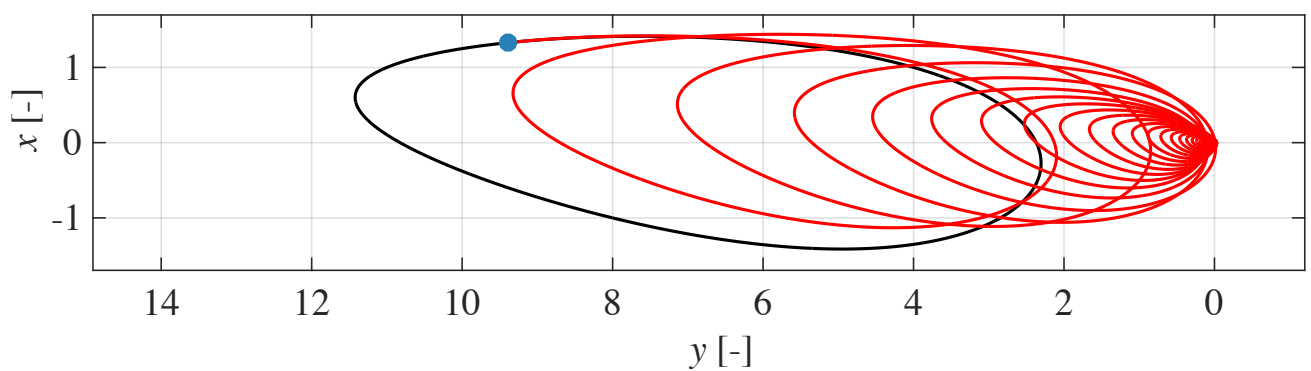

(c) $q=-4$.

Fig. 11 The optimal controlled trajectories departing from the optimal initial position for $q=3,0,-4$. 


\section{Conclusion}

This paper considered the optimal trajectory design for formation flying along an elliptic orbit. The attractive set of optimal reconfiguration problem has been proposed based on the linear quadratic regulator theory for the Tschauner-Hempel equations. The geometric approach to depict the attractive sets using periodic properties of the Tschauner-Hempel equations has been proposed. Then, the optimal initial state along an initial periodic orbit can be found as the tangent point between the periodic orbit of the chaser and the maximum attractive set inscribed in it. Simulation results on the shape of the attractive sets for different weight parameters revealed that the attractive sets reflect the dynamical structure of equations of motion. Especially, the attractive sets have an interesting property that their shapes converge to the shape of the initial periodic orbit of the chaser as the weight parameter of control input is increased.

\section{Acknowledgments}

The work of the second author was partially supported by JSPS KAKENHI Grant Number JP18K04560.

\section{References}

[1] Gurfil, P., "Relative motion between elliptic orbits: generalized boundedness conditions and optimal formationkeeping," Journal of Guidance, Control, and Dynamics, Vol. 28, No. 4, 2005, pp. 761-767. doi:10.2514/1.9439.

[2] Li, J., and Xi, X.-n., "Fuel-optimal low-thrust reconfiguration of formation-flying satellites via homotopic approach,” Journal of Guidance, Control, and Dynamics, Vol. 35, No. 6, 2012, pp. 1709-1717. doi:10.2514/1.57354.

[3] Clohessy, W., "Terminal guidance system for satellite rendezvous," Journal of the Aerospace Sciences, Vol. 27, No. 9, 1960, pp. 653-658. doi:10.2514/8.8704.

[4] Wie, B., Space Vehicle Dynamics and Control, AIAA, 1998, pp. 282-285. doi:10.2514/4.860119.

[5] Alfriend, K., Vadali, S. R., Gurfil, P., How, J., and Breger, L., Spacecraft formation flying: Dynamics, control and navigation, Vol. 2, Elsevier, 2009.

[6] Yamanaka, K., and Ankersen, F., "New state transition matrix for relative motion on an arbitrary elliptical orbit," Journal of guidance, control, and dynamics, Vol. 25, No. 1, 2002, pp. 60-66. doi:10.2514/2.4875.

[7] Sinclair, A., Sherrill, R., and Lovell, T., "Review of the solutions to the Tschauner-Hempel equations for satellite relative motion,” AIAA/AAS Space Flight Mechanics Meeting, Charleston, South Carolina, Vol. AAS 12-149, 2012.

[8] Sengupta, P., and Vadali, S. R., "Relative motion and the geometry of formations in Keplerian elliptic orbits with arbitrary eccentricity," Journal of Guidance, Control, and Dynamics, Vol. 30, No. 4, 2007, pp. 953-964. doi:10.2514/1.25941.

[9] Bando, M., and Ichikawa, A., "Graphical generation of periodic orbits of TschaunerHempel equations," Journal of Guidance, Control, and Dynamics, Vol. 35, No. 3, 2012, pp. 1002-1007. doi:10.2514/1.56326. 
[10] Redding, D. C., Adams, N. J., and Kubiak, E. T., "Linear-quadratic stationkeeping for the STS orbiter," Journal of Guidance, Control, and Dynamics, Vol. 12, No. 2, 1989, pp. 248-255. doi:10.2514/3.20398.

[11] Kapila, V., Sparks, A. G., Buffington, J. M., and Yan, Q., "Spacecraft formation flying: Dynamics and control," Journal of Guidance, Control, and Dynamics, Vol. 23, No. 3, 2000, pp. 561-564. doi:10.2514/2.4567.

[12] Kristiansen, R., and Nicklasson, P. J., "Spacecraft formation flying: a review and new results on state feedback control,” Acta Astronautica, Vol. 65, No. 11-12, 2009, pp. 1537-1552. doi:10.1016/j.actaastro.2009.04.014.

[13] Shibata, M., and Ichikawa, A., "Orbital Rendezvous and Flyaround based on Null Controllability with Vanishing Energy," Journal of Guidance, Control, and Dynamics, Vol. 30, No. 4, 2007, pp. 934-945. doi:10.2514/1.24171.

[14] Bando, M., and Scheeres, D. J., “Attractive Sets to Unstable Orbits Using Optimal Feedback Control,” Journal of Guidance, Control, and Dynamics, Vol. 39, No. 12, 2016, pp. 2725-2739. doi:10.2514/1.G000524.

[15] Yamane, M., Bando, M., and Hokamoto, S., "Optimal Trajectory Design of Formation Flying based on Attractive Sets," Transactions of the Japan Society for Aeronautcal and Space Sciences, Aerospace Technology Japan, Vol. 17, No. 1, 2019, pp. 96-103. doi:10.2322/tastj.17.96.

[16] Vaddi, S., Alfriend, K. T., Vadali, S., and Sengupta, P., "Formation establishment and reconfiguration using impulsive control," Journal of Guidance, Control, and Dynamics, Vol. 28, No. 2, 2005, pp. 262-268. doi:10.2514/1.6687.

[17] Ichikawa, A., and Katayama, H., Linear Time-Varying Systems and Sampled-Data Systems, Vol. 265, Springer-Verlag, London, 2001, pp 19-27. doi:10.1007/BFb0110391. 